\title{
Magnetically Induced Thermal Rectification
}

\author{
Giulio Casati, ${ }^{1,2,3}$ Carlos Mejía-Monasterio, ${ }^{4}$ and Tomaž Prosen ${ }^{5}$ \\ ${ }^{1}$ Center for Nonlinear and Complex Systems, Università degli Studi dell'Insubria, Como Italy \\ ${ }^{2}$ CNR-INFM and Istituto Nazionale di Fisica Nucleare, Sezione di Milano \\ ${ }^{3}$ Department of Physics, National University of Singapore, Republic of Singapore \\ ${ }^{4}$ Dipartimento di Matematica, Politecnico di Torino, Italy \\ ${ }^{5}$ Physics Department, Faculty of Mathematics and Physics, University of Ljubljana, Ljubljana, Slovenia
}

(Received 7 November 2006; published 8 March 2007)

\begin{abstract}
We consider far from equilibrium heat transport in chaotic billiard chains with noninteracting charged particles in the presence of nonuniform transverse magnetic field. If half of the chain is placed in a strong magnetic field, or if the strength of the magnetic field has a large gradient along the chain, heat current is shown to be asymmetric with respect to exchange of the temperatures of the heat baths. Thermal rectification factor can be arbitrarily large for sufficiently small temperature of one of the baths.
\end{abstract}

DOI: 10.1103/PhysRevLett.98.104302

The problem of explaining irreversible macroscopic transport laws, such as the Fourier law of heat conduction, from the reversible microscopic equations of motion is one of the open problems in nonequilibrium statistical mechanics $[1,2]$. The problem is still far from being settled. In systems of noninteracting particles, e.g., quasi 1-d chaotic billiards, the validity of Fourier law has clearly been confirmed [3], even though such noninteracting systems cannot exhibit local thermal equilibrium in the nonequilibrium steady state [4,5]. Furthermore, an interesting connection between anomalous heat conduction and anomalous diffusion has been established [6]. These problems are not only interesting for understanding the fundamentals of statistical mechanics, but they may also have straightforward applications, e.g., for connecting dynamics and transport in emerging nanotechnology, engineering of molecular motors, understanding and control of energy flow in biomolecules, etc.

In view of these ideas, Terraneo et al. [7] have recently proposed a mechanism for thermal rectification in an anharmonically interacting particle chain. Using an effective phonon approach, they have shown that an anharmonic chain composed of three different parts may have asymmetric heat transport properties due to (non)matching of the effective phonon bands. This and related ideas have been further elaborated and improved [8,9], achieving rectification efficiencies up to 2000 . Recently, the ideas in [8] have led to an interesting experimental work in which thermal rectification has been observed [10]. A further step to devise a thermal transistor has been discussed in [11] in terms of the negative differential thermal resistance observed in some anharmonic chains.

Since these first works, other different mechanisms leading to thermal rectification have been described. In [12], it was shown that the nonlinearity of the dynamics of an asymmetric two-level system leads to an asymmetric heat flow. More recently in [13], it was shown that thermal rectification can be observed in asymmetric billiards of interacting particles. When the billiard is subjected to an
PACS numbers: 44.10.+i, 05.45. $-\mathrm{a}$, 05.70.Ln

external temperature gradient, the effective interaction leads to a temperature dependence of the transmission coefficient, and thus, it is possible to dynamically control the transmission probability of the billiard. For this type of billiard system, rectifications as large as $10^{3}$ were observed. However, the theory presented in [13] requires the knowledge of the microscopic transmission coefficients, which is, at best, phenomenological. Simple phenomenological mechanism of thermal rectification has also been recently discussed in [14].

A common problem with most of these proposals is that the rectification factor has always been rather limited; i.e., it is very difficult to achieve a situation in which heat flows only in one direction and not in the opposite one. Moreover, the fact that the thermal rectification depends directly or indirectly on the microscopic particle-particle interaction renders difficult the ability to control the power of rectification.

In this Letter, we propose a novel microscopic mechanism for thermal rectification which works in the absence of particle interactions. Instead, thermal rectification is controlled with an external nonuniform magnetic field, leading to an arbitrarily large power of rectification. In our model, heat is carried by charged particles and the only restriction is that the typical mean-free-paths due to dissipative mechanisms should be much larger than the Larmor radius of the charged particles in the magnetic field. In practice, this means that the temperatures of the baths should be quite low, but such regimes are nowadays easily accessible, for example, in mesoscopic physics, quantum dots/wires, antidot lattices, etc. The rectification mechanism is very simple. It is based on asymmetric reflection of slow and fast particles off the interface between regions with different magnetic field intensities and predicts arbitrary large rectification factors for sufficiently low temperatures.

We consider a gas of noninteracting point particles of mass $m$ and electric charge $e$ that moves freely inside a closed two-dimensional billiard region. The billiard is 
shaped as a chain of equal cells as depicted in Fig. 1. Let the circular obstacles have radius $R$ and centers of the discs be arranged in a hexagonal array with lattice distance $4 / \sqrt{3}$, such that the billiard motion has a closed horizon for $R \geq 1$. Then we cut out a quasi one-dimensional billiard channel of width $\delta y=2 / \sqrt{3}$ through the centers of two nearby rows of discs so that one rectangular $\delta x \times \delta y$ cell of the channel, containing one half disk and two quarter disk obstacles, has length $\delta x=4$. Negative curvature of the billiard boundary ensures that the motion in the absence of the magnetic field is completely chaotichyperbolic. The simplest model that we consider is composed of two cells (Fig. 1). The left cell contains no magnetic field, whereas the right cell is subjected to a perpendicular uniform magnetic field of strength $B$. In what follows, we will refer to a channel of $N$ cells for which the $N / 2$ left cells contain no magnetic field and the $N / 2$ right cells contain a magnetic field of density $B$, as step configuration. In the dynamics, we neglect the Coulomb interaction among particles. Therefore, our results are valid for sufficiently low particle density. Let $\lambda$ denote the smallest length scale in the problem, namely, the width of the opening between the neighboring cells in our model, $\lambda=\frac{2}{\sqrt{3}}-R$.

The transmission probability between the two cells is controlled by the strength of the magnetic field. Consider the particles that cross the interface from left to right. There exists a critical velocity $v_{c}$ : fast particles of velocity $v>v_{c}$ always enter the right cell, and thus contribute to the left to right energy flow provided they reach the right end of the system which is coupled to a heat bath as explained below. Instead, slow particles of velocity $v<$ $v_{c}$, such that the gyro-magnetic radius $\varrho(v)=m v /(e B)$ is less than $\lambda / 2$, will be reflected or transmitted depending on the position at which they reach the interface.

Using a statistical ensemble of trajectories, the condition for the critical velocity $\varrho\left(v_{c}\right)=\lambda / 2$ can be rewritten as the condition giving a critical temperature

$$
T_{c}=\frac{\left(e B_{c} \lambda\right)^{2}}{8 m k_{B}}
$$

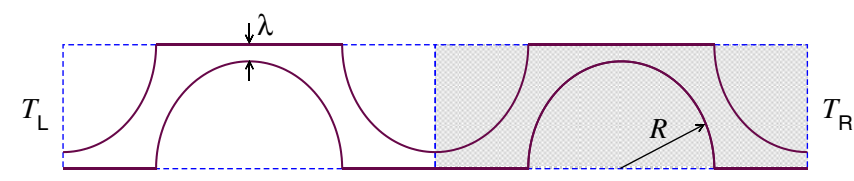

FIG. 1 (color online). Geometry of the model: We consider a closed billiard composed by $N$ symmetric unit cells, each of which is made of a rectangular boundary of width $\delta y=2 / \sqrt{3}$ and length $\delta x=4$. Inside each cell, there are three circular hard scatterers of radius $R$ disposed in a triangular lattice (see the text). Each cell is subjected to an external perpendicular uniform magnetic field of strength $B_{i} \in[0, B]$. At the left and right boundaries, the billiard is placed in contact with two stochastic thermal baths at different temperatures $T_{L}$ and $T_{R}$. The figure corresponds to a channel of $N=2$ cells in which $B_{1}=0$ and $B_{2}=B$. The dashed lines are drawn as a reference. such that particles which are colder than $T_{c}$ will be reflected in their majority.

However, for the particles that cross the interface from right to left, there is no condition on their velocity, and they always enter the left cell. The above qualitative argument makes it clear that the transport of heat will be strongly asymmetric with respect to exchange of effective temperatures of particles on different sides of magnetic field boundary, provided the temperatures are strongly different, one being larger and the other smaller than $T_{c}$. In the rest of the Letter, we measure the temperature in units of $T_{c}$ and denote it as $\tau=T / T_{c}=\frac{m}{2 k_{B} T_{c}}\left\langle v^{2}\right\rangle$.

We couple the left cell with a stochastic heat bath [3] of dimensionless temperature $\tau_{L}$ and the right cell with a heat bath of temperature $\tau_{R}$, namely, if a particle reaches the end boundary of the billiard that is in contact with a heat bath, it is reflected with a velocity chosen from a distribution with probability densities

$$
P_{\perp}\left(v_{x}\right)=\frac{\left|v_{x}\right|}{\tau} e^{-v_{x}^{2} / 2 \tau}, \quad P_{\|}\left(v_{y}\right)=\frac{1}{\sqrt{2 \pi \tau}} e^{-v_{y}^{2} / 2 \tau},
$$

where $\tau$ is the temperature of the respective heat bath in dimensionless units. If the left bath is cold, i.e., $\tau_{L} \ll 1$, then the particles will most of the time remain in the left cell, and there will be no heat current between the baths, irrespective of the temperature of the right bath, which we assume is larger than 1. If we exchange the temperatures of the baths, then we will in general have some nonsmall heat current flowing, since cold particles have no problem in leaving the region with a magnetic field.

We measure the heat current per particle in the steady state as the time average of the energy transported across the junction per unit time

$$
J\left(x_{0}\right)=\lim _{t \rightarrow \infty} \frac{1}{t} \int_{0}^{t} E\left(t^{\prime}\right) \operatorname{sgn}\left[v_{x}\left(t^{\prime}\right)\right] \delta\left(x\left(t^{\prime}\right)-x_{0}\right) d t^{\prime},
$$

where $E(t)=\frac{1}{2} m\left[v_{x}^{2}(t)+v_{y}^{2}(t)\right]$ is the instantaneous kinetic energy. Furthermore, we denote the heat current as $J^{+}$if $\tau_{L}<\tau_{R}$ and as $J^{-}$if the temperatures are exchanged, i.e., $\tau_{L}>\tau_{R}$. Comparing magnitudes of these two currents, we quantify the thermal rectification as

$$
\Delta=\frac{\max \left\{\left|J^{+}\right|,\left|J^{-}\right|\right\}}{\min \left\{\left|J^{+}\right|,\left|J^{-}\right|\right\}} .
$$

From our argument, it is clear that rectification will be effective if one of the temperatures is very small, say $\tau_{L} \ll$ 1 , and the other is simply above the critical, $\tau_{R}>1$. It is possible to make a quantitative prediction on the scaling of rectification factor $\Delta$ with temperatures. When the energy current through the magnetic field interface is very weak, namely, if $\tau_{L}<\tau_{R}$, the current is proportional to the transmission coefficient at the interface, i.e., one minus the probability of reflection. From our previous discussion, it is clear that if $\tau_{L}<1<\tau_{R}$, the particle density will be larger at the left cell (with zero magnetic field). This is mainly because the cold particles in the left cell spend a 
long time before being able to cross the interface. A particle of velocity $v$ is transmitted (not reflected) to the right cell if it crosses the interface at a distance from the upper boundary shorter than $2 \varrho(v)$ [15]. Therefore, invoking the ergodicity of the dynamics in the left cell, we can simply estimate the transmission coefficient $t$ as $t^{+} \sim$ $\frac{2 \varrho(v)}{\lambda}$, where $\varrho(v(\tau))=\sqrt{2 m k_{B} \tau_{\min }} / e B$ and we denote by $\tau_{\min }=\min \left\{\tau_{L}, \tau_{R}\right\}$. However, in the reverse situation (exchanging $\tau_{L}$ and $\tau_{R}$ ), we have $t^{-} \sim 1$, so we can estimate the rectification

$$
\Delta=t^{-} / t^{+} \propto \frac{1}{\sqrt{\tau_{\min }}} .
$$

In Fig. 2, we show the rectification index $\Delta$ as a function of $\tau_{\min }$ for fixed value of the maximal temperature $\tau_{\max }$. We clearly confirm the scaling (4), indicating also that the rectification index is only very weakly depending on the maximal temperature (as long as $\tau_{\max }>1$. This can be seen in the inset of Fig. 2) where the rectification index $\Delta$ is shown as a function of the two temperatures of the heat baths $\tau_{L}$ and $\tau_{R}$. The correctness of the scaling (4) shows that the magnetically induced rectification power is arbitrarily large for sufficiently small temperature $\tau_{\min }$. Even though a noninteracting system cannot reach local thermal equilibrium, and therefore the concept of (local) temperature is not well defined, it is instructive to compute temperature and density profiles, as long-time averages of kinetic energy density and particle density as a function of the horizontal coordinate along the chain. In Fig. 3, we plot the kinetic energy density and the particle density measured in each cell of a channel of 10 cells, the right half of 5 cells being in a uniform magnetic field. We plot the profiles for the positive gradient, $\tau_{L}<\tau_{R}$ (triangles), and the inverted negative gradient (circles). The positive

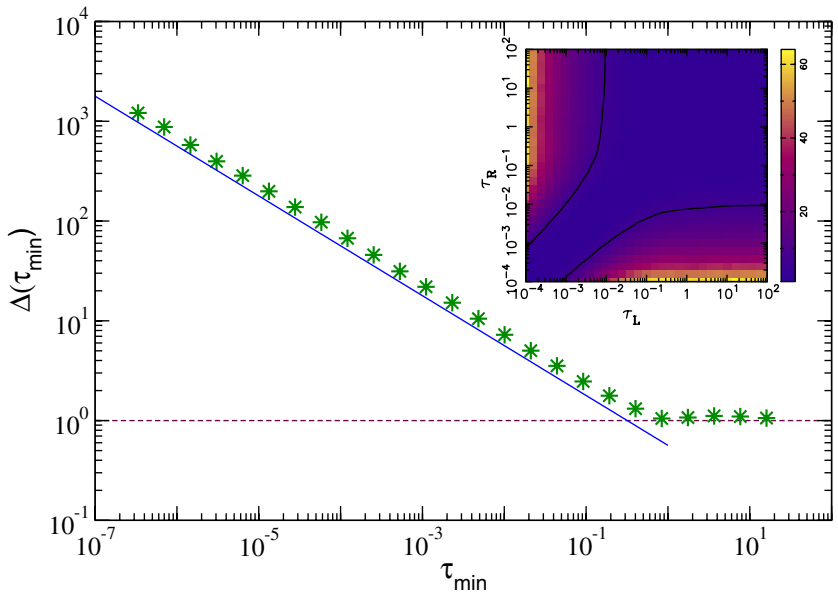

FIG. 2 (color online). Rectification index $\Delta$ as a function of the minimal temperature $\tau_{\min }$ for a 2-cell channel in a step configuration. The maximal temperature was set to $\tau_{\max }=33.4275$. The dashed line is for the no rectification value $\Delta=1$. The solid line corresponds to $\tau_{\min }^{-1 / 2}$. Inset: Color density plot for $\Delta$ as a function of the temperatures of both baths. gradient for which $\tau_{L}$ is $\tau_{\min }$ corresponds to the insulating case, i.e., to the situation in which the heat current is very small. In Fig. 3, we see that the insulating case corresponds to a large gap in the kinetic energy profile. In contrast, for the negative gradient, the gap in the kinetic energy profile is much smaller, and this coincides with the observation of a larger heat current. However, note that the energy profile for the negative gradient is not a monotonous function due to the lack of local thermal equilibrium. Moreover, the density profile confirms our prediction that the low current situation is characterized by a very small density of particles on the side of the magnetic field.

We discuss now a slightly different situation in which the magnetic field does not change abruptly from one half of the system to the other, but instead changes gradually, forming a uniform gradient of the magnetic field. We refer to this situation as the gradient configuration for which the magnetic field in each cell has an intensity given by $B_{i}=$ $B(i-1) /(N-1)$ for $i=1,2, \ldots, N$.

We have performed numerical simulations for the gradient configuration, and we found no qualitative differences with respect to the step configuration. In Fig. 4, we show the dependence of the rectification index on the minimal temperature (panel $a$ ). As for the step configuration, we have found that for sufficiently low temperature $\tau_{\min }$, the rectification index again grows as $\Delta \sim 1 / \sqrt{\tau_{\min }}$. In the panels $(b)$ and $(c)$, we show the profiles of the kinetic energy and particle density, respectively. As expected, the profiles for the gradient configuration are more smooth than for the step configuration.

From our analysis, it follows that our rectification effect is a phenomenon which exists only in far from equilibrium situation. In the thermodynamic limit, a vanishingly small temperature gradient is established across the system. In Fig. 5, we show the results of numerical simulations which indicate that, for fixed bath temperatures, the rectification index scales as $\Delta \sim 1 / L$. In the inset of Fig. 5 , the time averaged heat currents for the positive and negative gradients are also shown.
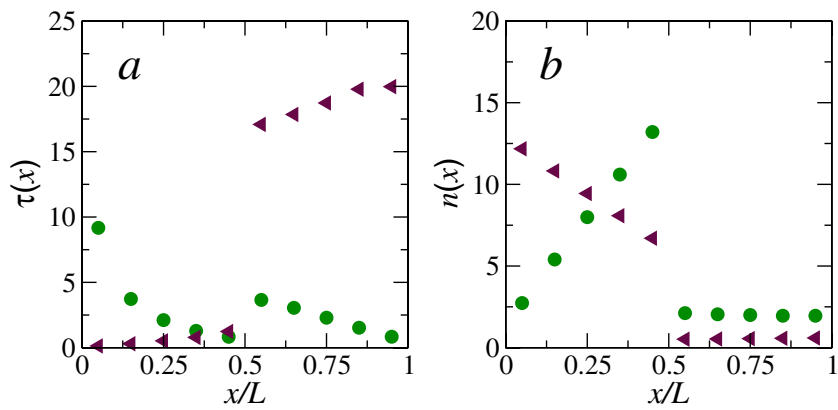

FIG. 3 (color online). Energy density (a) and particle density (b) profiles for a 10-cell channel in a step configuration. The bath temperatures are $\tau_{\min }=3.34277 \times 10^{-2}, \tau_{\max }=33.4277$. In both panels, profiles are shown for the positive and negative gradient for which $\tau_{R}=\tau_{\max }$ (triangles) and $\tau_{R}=\tau_{\min }$ (circles), respectively. 

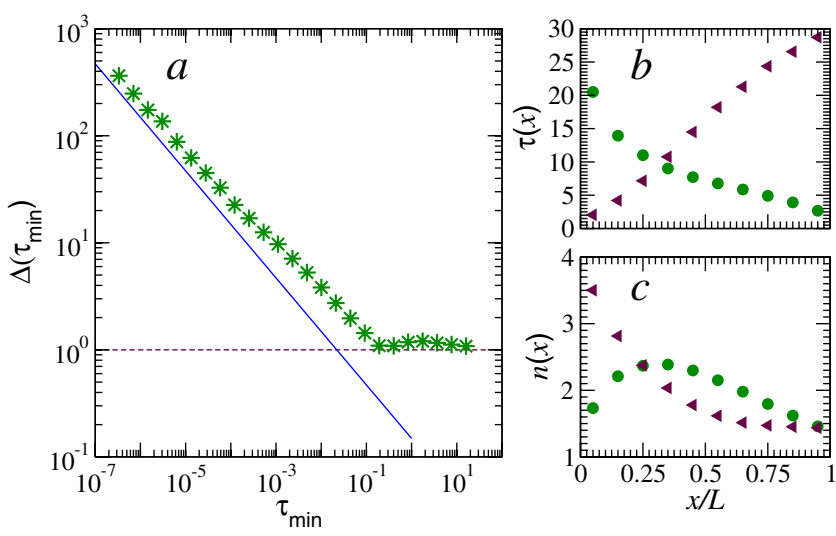

FIG. 4 (color online). 10-cell channel in a gradient configuration: (a) Rectification index $\Delta$ as a function of the minimal temperature $\tau_{\min }$, with $\tau_{\max }=33.4275$. The temperatures are given in units of $T_{c}(B)$, where $B=100$ is the strength of the magnetic field at the rightmost cell. The dashed line is for the no rectification value $\Delta=1$. The solid line corresponds to $\tau_{\min }^{-1 / 2}$. In the panels at the right, the profiles of kinetic energy (b) and particle density (c) for the positive (triangles) and negative (circles) gradient are shown.

Finally, it is interesting to give a quantitative estimate of the critical temperature in Eq. (1) in physical units. Let us suppose that the gas of particles inside the billiard of Fig. 1 consists of electrons. Assuming that the dimension of the opening is $\lambda=100 \mathrm{~nm}$ and a magnetic field of $B=1 \mathrm{~T}$ is applied, then the critical temperature is $T_{c} \sim 0.5 \mathrm{~K}$. Thus, a rectification power of $\Delta \sim 10$ would be measurable for thermal gradient given by $T_{\min } \sim 10^{-3} \mathrm{~K}$ and $T_{\max } \sim 10 \mathrm{~K}$ that appears accessible to nowadays experiments.

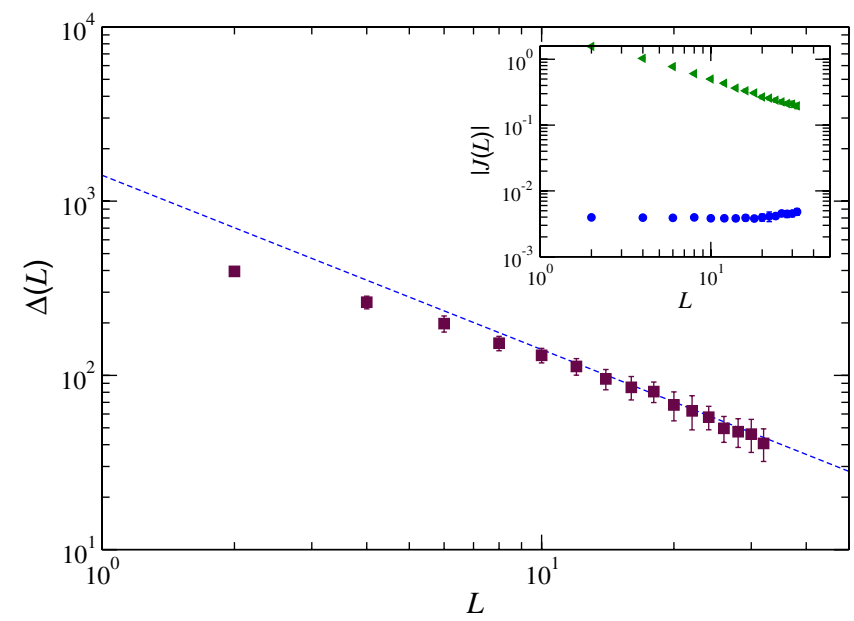

FIG. 5 (color online). Dependence of the thermal rectification index $\Delta$ as a function of the size of the chain $L$ for a chain in a step-configuration with fixed $\tau_{\min }=3.34275 \times 10^{-3}, \tau_{\max }=$ $3.34275 \times 10^{2}$ and fixed particle density, namely, one particle per cell. The dashed line corresponds to $L^{-1}$. Inset: heat current $J$ as a function of the size of the chain $L$ for the positive (triangles) and negative (circles) gradient.
In this Letter, we have presented a novel mechanism for thermal rectification. This mechanism is fairly simple. It results from the asymmetric behavior of the dynamics at the magnetic interface that leads to a simple temperature dependence of the transmission coefficient. Moreover, this mechanism for thermal rectification is not based on the macroscopically unaccessible microscopic particle interaction, but on the interaction with an external field, making possible an easy control of the power of rectification. We have shown that the thermal rectification power is arbitrarily large for sufficiently small temperature of one of the heat baths.

Furthermore, the physical scales needed for optimal implementation of our theoretical model are realizable in present nanoscale experiments with mesoscopic devices. We believe that it is precisely at these scales of mesoscopic physics where such a thermal rectifier would find interesting applications. This fact makes an experimental verification of the mechanism presented here very desirable.

We acknowledge support by the Institute for Scientific Interchange (C. M. M.), Grant MIUR-PRIN 2005 (G. C.), and Grants P1-0044 and J1-7347 of Slovenian Research Agency (T. P.).

[1] F. Bonetto, J. L. Lebowitz, and L. Rey-Bellet, in Mathematical Physics 2000 (Imp. Coll. Press, London, 2000) pp. 128.

[2] S. Lepri, R. Livi, and A. Politi, Phys. Rep. 377, 1 (2003).

[3] D. Alonso, R. Artuso, G. Casati, and I. Guarneri, Phys. Rev. Lett. 82, 1859 (1999).

[4] A. Dhar and D. Dhar, Phys. Rev. Lett. 82, 480 (1999).

[5] C. Mejia-Monasterio, H. Larralde, and F. Leyvraz, Phys. Rev. Lett. 86, 5417 (2001); H. Larralde, F. Leyvraz, and C. Mejia-Monasterio, J. Stat. Phys. 113, 197 (2003).

[6] B. Li and J. Wang, Phys. Rev. Lett. 91, 044301 (2003); B. Li, J. Wang, L. Wang, and G. Zhang, Chaos 15, 015121 (2005).

[7] M. Terraneo, M. Peyrard, and G. Casati, Phys. Rev. Lett. 88, 094302 (2002).

[8] B. Li, L. Wang, and G. Casati, Phys. Rev. Lett. 93, 184301 (2004).

[9] B. Li, J.-H. Lan, and L. Wang, Phys. Rev. Lett. 95, 104302 (2005).

[10] C. W. Chang, D. Okawa, A. Majumdar, and A. Zettl Science 314, 1121 (2006).

[11] B. Li, L. Wang, and G. Casati, Appl. Phys. Lett. 88, 143501 (2006).

[12] D. Segal and A. Nitzan, Phys. Rev. Lett. 94, 034301 (2005).

[13] J.-P. Eckmann and C. Mejia-Monasterio Phys. Rev. Lett. 97, 094301 (2006).

[14] M. Peyrard, Europhys. Lett. 76, 49 (2006); B. Hu, D. He, L. Yang, and Y. Zhang, Phys. Rev. E 74, 060201(R) (2006).

[15] The particles that are transmitted to the right cell bounce repeatedly with either the upper or the lower boundary of the billiard and this depends on the sign of the magnetic field. 